\title{
APLICAÇÃo DE MÉTODOS DE CALIBRAÇÃo MULTIVARIADA PARA A DETERMINAÇÃo SIMULTÂNEA DE RIBOFLAVINA (VB2), TIAMINA (VB1), PIRIDOXINA (VB6) E NICOTINAMIDA (VPP)
}

\author{
Rosângela C. Barthus* e Luiz H. Mazo \\ Instituto de Química de São Carlos, Universidade de São Paulo, CP 780, 13560-970 São Carlos - SP, Brasil \\ Ronei J. Poppi \\ Departamento de Química Analítica, Instituto de Química, Universidade Estadual de Campinas, CP 6154, 13084-971 \\ Campinas - SP, Brasil
}

Recebido em 20/9/06; Aceito em 23/3/07; publicado na web em 6/8/07

\begin{abstract}
UV SPECTROPHOTROMETRY AND CHEMOMETRICS METHODS FOR SIMULTANEOUS DETERMINATIONS OF RIBOFLAVIN (VB2), THIAMINE (VB1), PYRIDOXINE (VB6) AND NICOTINAMIDE (VPP). In this work, the artificial neural networks (ANN) and partial least squares (PLS) regression were applied to UV spectral data for quantitative determination of thiamin hydrochloride (VB1), riboflavin phosphate (VB2), pyridoxine hydrochloride (VB6) and nicotinamide (VPP) in pharmaceutical samples. For calibration purposes, commercial samples in $0.2 \mathrm{~mol} \mathrm{~L}^{-1}$ acetate buffer $(\mathrm{pH} 4.0)$ were employed as standards. The concentration ranges used in the calibration step were: $0.1-7.5 \mathrm{mg} \mathrm{L}^{-1}$ for VB1, $0.1-3.0 \mathrm{mg} \mathrm{L}^{-1}$ for VB2, $0.1-3.0 \mathrm{mg} \mathrm{L}^{-1}$ for VB6 and $0.4-30.0 \mathrm{mg} \mathrm{L}^{-1}$ for VPP. From the results it is possible to verify that both methods can be successfully applied for these determinations. The similar error values were obtained by using neural network or PLS methods. The proposed methodology is simple, rapid and can be easily used in quality control laboratories.
\end{abstract}

Keywords: water-soluble vitamins; UV spectrophotometry; multivariate calibration.

\section{INTRODUÇÃO}

Vitaminas são substâncias orgânicas indispensáveis à manutenção das funções metabólicas do organismo. A maior parte das vitaminas atua como coenzima; isto explica seu papel essencial nos processos biológicos. Essas substâncias apesar de essenciais ao metabolismo, não são sintetizadas pelo organismo e, em virtude disso, necessitam ser adquiridas de outras formas ${ }^{1}$. Em dias atuais, alimentos enriquecidos e medicamentos estão se tornando importantes meios para a obtenção de vitaminas para o nosso organismo. Dessa forma, métodos que assegurem a qualidade destes produtos são essenciais. A maioria dos métodos descritos na literatura envolve separações químicas e físicas ${ }^{2-5}$. Dentre esses métodos, incluem-se os de cromatografia líquida de alta eficiência (CLAE) e os que envolvem titulação. As principais desvantagens destes métodos consistem em um relativo alto custo, emprego de reagentes tóxicos e, em muitos casos, um grande consumo de tempo para a execução das análises. Desde a ultima década, contudo, o uso de métodos espectroscópicos aliados à calibração multivariada tem-se mostrado um meio rápido e eficiente para a determinação de diversos tipos de substâncias em amostras farmacêuticas. Algumas publicações mostram o uso de técnicas espectrofotométricas e eletroquímicas em conjunto com métodos de calibração multivariada para a determinação de vitaminas ${ }^{6-}$ ${ }^{10}$. Em muitos trabalhos, amostras sintéticas foram utilizadas na fase de calibração. Nestes casos, a maioria das amostras utilizadas apresenta menor complexidade e embora nenhum pré-tratamento mais rigoroso seja aplicado, muitas vezes são realizadas separações prévias dos excipientes. Este tipo de determinação não se aplica para amostras mais complexas.

*e-mail: rcbarthus@hotmail.com
O principal objetivo deste trabalho foi verificar a aplicação de métodos de calibração multivariada na determinação de vitaminas hidrossolúveis quando existe uma grande variação dos constituintes (excipientes, quantidade de substâncias e mesmo outros tipos de vitaminas) nas amostras analisadas. Devido à complexidade e variedade entre as amostras é necessário empregar amostras reais na calibração para a obtenção de um modelo representativo.

No presente estudo, dois métodos de calibração para a determinação de vitaminas hidrossolúveis foram avaliados usando a técnica de espectrofotometria na região do ultravioleta: Redes neurais artificiais (RNAs) e mínimos quadrados parciais (PLS).

\section{Teoria}

Mínimos quadrados parciais (PLS) ${ }^{11-13}$ é o método normalmente utilizado em análises de calibração multivariada. Neste método, os sinais multivariados, como os valores de absorbâncias medidos em diferentes comprimentos de onda do espectro na região do ultravioleta (variáveis $x$ ) e concentrações das vitaminas nas amostras (variáveis y), são utilizados para estabelecer um modelo de regressão linear. Para a construção do modelo de calibração, os dados são dispostos na forma de matrizes: Matriz X e Matriz Y que contêm os dados de variáveis independentes $\mathrm{x}$ e dependentes $\mathrm{y}$, respectivamente. Estas matrizes são decompostas em uma soma de produtos de dois vetores, os "scores" e os "loadings", obtendose os seguintes modelos:

$\mathrm{X}=\mathrm{TP}^{\mathrm{t}}+\mathrm{E}=\Sigma \mathrm{t}_{\mathrm{f}} \mathrm{p}_{\mathrm{f}}^{\mathrm{t}}+\mathrm{E}$

$\mathrm{Y}=\mathrm{TQ} \mathrm{Q}^{\mathrm{t}}+\mathrm{F}=\Sigma \mathrm{t}_{\mathrm{f}} \mathrm{f}_{\mathrm{f}}^{\mathrm{t}}+\mathrm{F}$

onde T corresponde à matriz de "scores", $\mathrm{P}$ e Q são os "loadings" para $\mathrm{X}$ e Y, respectivamente e E, F representam os erros, ou falta de ajuste 
do modelo. O sobrescrito $t$ indica a matriz transposta. Uma relação entre os dois blocos (variáveis independentes $\mathrm{X}$ e variáveis dependentes $\mathrm{Y}$ ) pode ser realizada correlacionando-se os "scores" dos blocos X e Y. Para que se encontre melhor correlação possível, torna-se importante manipular a informação desses dois blocos simultaneamente. No PLS isto é feito por uma leve rotação das componentes principais (devido a esta rotação, são chamadas agora de variáveis latentes), com conseqüente mudança nos valores dos "scores". O número de variáveis latentes utilizado no processo de calibração é obtido por meio de validação cruzada, que é uma metodologia baseada na magnitude dos erros de previsão de um dado modelo de calibração. Depois de estabelecidos o número de variáveis latentes e o modelo, este pode ser destinado à previsão de concentração de novas amostras. A concentração de novas amostras pode ser estimada a partir dos novos "scores" $\mathrm{T}^{*} \mathrm{e}$ dos "loadings" Q do modelo de calibração, que podem ser substituídos na equação 2 , obtendo-se a equação 3 .

$\mathrm{Y}_{\mathrm{n}}=\mathrm{T}^{*} \mathrm{Q}^{\mathrm{t}}$

Normalmente o PLS assume uma relação linear entre os parâmetros analisados. Pequenos desvios da linearidade são aceitáveis, ou seja, o modelo é capaz de dar uma boa previsão desde que mais variáveis latentes sejam incorporadas ao modelo. Contudo, quando substancial não-linearidade está presente nos dados analisados, erros consideráveis são obtidos. Nestes casos, modelos de calibração não-lineares como as redes neurais artificiais ${ }^{14-16}$ são mais adequados.

Redes neurais artificiais (RNAs) é o nome dado a um conjunto de métodos matemáticos e algoritmos computacionais especialmente projetados para simular o processamento de informações e a aquisição de conhecimentos do cérebro humano. A rede realiza este trabalho "aprendendo" como o sistema em estudo se comporta e, então, aplica este conhecimento a novos dados de entrada para predizer a saída desejada. Este método de calibração multivariada é empregado principalmente na análise de dados não-lineares, embora algumas aplicações em casos de dados lineares possam ser encontradas. Uma das principais vantagens em relação aos métodos paramétricos é sua maior flexibilidade para o tratamento dos dados e a obtenção de um modelo de calibração.

A rede neural artificial é um sistema composto de várias unidades simples (neurônios artificiais), propriamente ligadas, produzindo um comportamento complexo. A estrutura da rede utilizada neste estudo é composta de uma primeira camada (neurônios de entrada), consistindo de dados de entrada, que correspondem às medidas de absorbância em diversos comprimentos de onda, ou ainda, dos valores de scores obtidos a partir da aplicação da análise de componentes principais aos dados de espectros. Esta camada de entrada é seguida por uma camada interna, com um número apropriado de neurônios, e esta por uma camada de saída (neurônios de saída) ou respostas das redes neurais correspondendo às concentrações das vitaminas nos medicamentos.

Para treinar a rede neural, os dados de entrada $\mathrm{x}$ multiplicados por seus pesos w são integrados em um neurônio artificial. A função $N e t=\Sigma \mathrm{x}_{\mathrm{i}} \mathrm{w}_{\mathrm{ij}}$ (4) é a somatória de todos os dados de entrada $\mathrm{x}$ vezes seus respectivos pesos w. A saída de cada camada é obtida pela aplicação de uma função de transferência, que pode ser sigmoidal, tangente hiperbólica, linear, entre outras. Normalmente um bias é adicionado à função de transferência para um melhor ajuste. Para redes neurais de múltiplas camadas os dados de saída de uma camada correspondem aos dados de entrada da camada seguinte. E os dados de saída da última camada correspondem às respostas da rede neural. A resposta $y_{p}$ da rede neural para um vetor de entrada $x$ pode ser escrita como mostrada na Equação 5, para uma rede com uma camada interna e um neurônio na camada de saída.
$y_{p}=\sum_{j=1}^{n_{H}} w_{j} h\left(\sum_{i=1}^{n_{1}} w_{j i} x_{i}+b_{j}\right)+B$

onde $h$ é uma função de transferência (sigmoidal, neste estudo), $b_{j}$ e $B$ representam os Bias do modelo, $w_{i j}$ é o valor do peso entre a camada de entrada e a camada interna e $w_{i}$ é o valor de peso entre a camada interna e a camada de saída, $n_{H}$ corresponde ao número de neurônios na camada interna e $n_{i}$ corresponde ao número de neurônio da camada de entrada.

A saída da rede neural $Y_{p}$ ou os valores estimados são comparados aos valores esperados $\mathrm{Y}$ obtendo-se o erro médio quadrático (erro de calibração). O erro é definido como a soma dos quadrados resultantes da diferença entre o valor estimado e o valor esperado.

$e=\sum_{i=1}^{N}\left(y-y_{p}\right)^{2}$

O próximo passo consiste em corrigir os pesos de todas as camadas até que o erro de calibração seja minimizado, o que é realizado por um algoritmo específico. O algoritmo utilizado para corrigir os pesos e os bias neste estudo foi de Marquardt-Levenberg, representado pela Equação 7:

$\Delta w=\left(\mathrm{J}^{\mathrm{t}} \mathrm{J}+\lambda \mathbf{l}\right)^{-1} \mathrm{~J}^{T} e$

onde $\mathrm{J}$ é a matriz jacobiana dos erros para cada peso, $\lambda$ é um escalar não negativo, I é a matriz identidade e $e$ representa um vetor de erros. Este método de aprendizado pode ser visto como um procedimento intermediário entre o método de Gauss-Newton e o método de gradiente descendente. Neste algoritmo, quando $\lambda$ assume elevados valores o método de gradiente descendente predomina e quando $\lambda$ assume pequenos valores o método de Gauss-Newton predomina. $\mathrm{O}$ método de Marquardt-Levenberg é mais rápido na convergência que outros algoritmos.

Depois de se corrigir os pesos e os bias e obter um erro satisfatório, a rede neural artificial está completamente treinada e, então, é possível avaliar a potencialidade do método de calibração desenvolvido para a determinação da concentração das vitaminas (no caso deste estudo) em um novo grupo de amostras.

\section{PARTE EXPERIMENTAL}

\section{Reagentes}

Todos os reagentes foram de grau analítico e utilizados sem tratamento de purificação prévia. Os reagentes cloridrato de tiamina (VB1), fosfato de riboflavina (VB2), cloridrato de piridoxina (VB6) e nicotinamida (VPP) foram de procedência da Sigma Chemical Co. O metanol utilizado foi de grau analítico para HPLC. Diidrogenofosfato de sódio ou fosfato ácido de sódio (Merck) e ácido fosfórico (Merck) foram utilizados para preparar solução tampão $0,05 \mathrm{~mol} \mathrm{~L}^{-1}, \mathrm{pH} 2,8$. Acetato de sódio e ácido acético foram utilizados para preparar solução tampão $0,2 \mathrm{~mol} \mathrm{~L}^{-1} \mathrm{e} \mathrm{pH} 4,0$. Solução de diidrogenofosfato de potássio $0,05 \mathrm{~mol} \mathrm{~L}^{-1}$ e $\mathrm{pH}$ 4,5 também foram utilizadas nos experimentos.

Água deionizada e purificada obtida a partir de um sistema Milli-Q (Millipore, Bedford, Ma, USA) foi utilizada em todos os procedimentos.

\section{Amostras farmacêuticas}

Os medicamentos utilizados nas análises apresentavam a seguinte composição: Complexo B ${ }^{\circledR}$ (EMS, Brasil) xarope: 30,0 mg de VB1, 
10,0 mg de VB2, 10,0 mg de VB6, 15,0 mcg de VB12, 25,0 mg de VPP, 15,0 mg pantetonato de cálcio e metilparabeno, propilparabeno, essência de caramelo, $\mathrm{Na}_{2}$ EDTA, colorante vermelho bordeaux, corante cor caramelo, álcool extra neutro e água de osmose como excipientes por $10 \mathrm{~mL}$, Blepexeron ${ }^{\circledR}$ (Ariston, Brasil): $10,0 \mathrm{mg}$ de VB1, 2,5 mg de VB2, 30,0 mg de VPP, 2,5 mg de VB6, 5,0 mg de pantetonato de cálcio e água esterilizada como excipientes por 2 $\mathrm{mL}$, Complexo $\mathrm{B}^{\circledR}$ (Roche, Brasil) gotas: 5,0 mg de VB1, 2,0 mg de VB2, 2,0 mg de VB6, 20,0 mg de VPP, 3,0 mg de VB5 (pantetonato de cálcio, D-pantenol), 1,25 mg de Biotina (vitamin H) essência de caramelo, aroma de nozes, glicerol, sacarina sódica e hidróxido de sódio como excipientes por $1 \mathrm{~mL}$, Complevitam ${ }^{\circledR}-1$ (Bunker, Brasil) xarope: 5,0 mg de VB1, 1,0 mg de VB2, 3,0 mg de VB6, 30,0 mg de VPP, 4,0 mg de pantetonato de cálcio, essência de abacaxi, ciclamato de sódio, sacarina, nipazol, nipagin, ácido cítrico, glicerina, como excipientes por $15 \mathrm{~mL}$, Suplevit ${ }^{\circledR}$ (EMS, Brasil) xarope, 2000 U de Vitamina A, 1,30 mg de VB1, 1,30 mg de VB2, 2,0 mg de VB6, 6,0 mcg de VB12, $400 \mathrm{U}$ de vitamina D, 10,0 mg de D-pantenol, 13,0 mg de VPP e ácido cítrico, polissorbato 80, sorbato de potássio, EDTA, BHT, essência de laranja, sacarina sódica, ciclamato de sódio, hidroxietilcelulose e água desmineralizada como excipiente por 5 $\mathrm{mL}$, Complevitam ${ }^{\circledR}-2$ (Bunker, Brasil) gotas: 3,0 mg de VB1, 3,0 mg de VB2, 3,0 mg de VB6, 10,0 mg de VPP, 25,0 mg de pantetonato de cálcio, essência de abacaxi, ciclamato de sódio, sacarina, nipazol, nipagin, ácido cítrico, glicerina, açúcar e sorbitol como excipientes por $5 \mathrm{~mL}$ e preparação manipulada, $2000 \mathrm{U}$ de Vitamina A, 1,30 mg de VB1, 1,30 mg de VB2, 2,0 mg de VB6, 6,0 mcg de VB12, $400 \mathrm{U}$ de vitamina D, 10,0 mg de D-pantenol, 13,0 mg de VPP e ácido cítrico, EDTA, sacarina sódica, ciclamato de sódio e água desmineralizada como excipientes por $5 \mathrm{~mL}$.

Um total de 14 amostras (duas de cada tipo) foi adquirido e utilizado para fins de calibração e previsão das concentrações das vitaminas nos medicamentos.

\section{Análise de cromatografia líquida de alta eficiência (CLAE)}

As amostras de medicamentos foram analisadas utilizando-se a técnica de cromatografia líquida de alta eficiência (CLAE) como uma técnica de referência. Para as análises foi utilizado um cromatógrafo Shimadzu (Tókio, Japão) equipado com bomba LC$10 \mathrm{AD}$, um detector SPD-M10A e um injetor universal. O software utilizado para o processamento dos dados foi o Class-LC, versão 1.64. Duas diferentes colunas cromatográficas foram utilizadas: uma coluna C18 (150 X 4,6 mm, $5 \mu \mathrm{m}$ ) (CGS- Instrumentos Científicos). A fase móvel do sistema consistiu de $\mathrm{KH}_{2} \mathrm{PO}_{4} 0,05 \mathrm{~mol} \mathrm{~L}$ ${ }^{1}$ ( $\left.\mathrm{pH} 4,5\right)$ - metanol $(75: 25, \mathrm{v} / \mathrm{v})$. A segunda coluna utilizada foi a Shim-Pack NH2 (150 X 6,0 mm) (Shimadzu). Com esta coluna, as separações das vitaminas foram feitas por eluição com solução tampão fosfato $0,05 \mathrm{~mol} \mathrm{~L}^{-1}(\mathrm{pH} \mathrm{2,8)}$ - metanol, (96:4, v/v) a um fluxo

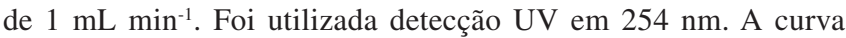
analítica foi obtida usando-se calibração externa ou seja, usando amostras padrões sintéticas das vitaminas. As soluções padrões utilizadas na calibração foram preparadas diariamente pela apropriada diluição das soluções estoques dos padrões.

Os medicamentos analisados, a concentração indicada no rótulo e as determinadas pela técnica CLAE são mostradas na Tabela 1.

\section{Amostras utilizadas na calibração e validação dos dados multivariados}

Para propostas de calibração multivariada um total de 140 amostras foram obtidas pela diluição apropriada das soluções estoques de sete preparações farmacêuticas, um de cada tipo em uma solução tampão acetato $0,2 \mathrm{~mol} \mathrm{~L}^{-1}$ e $\mathrm{pH} 4,0$. A faixa final de concentração utilizada neste estudo foi de 0,4 a $30,0 \mathrm{mg} \mathrm{L}^{-1}$ para a nicotinamida (VPP), 0,1 a 3,0 $\mathrm{mg} \mathrm{L}^{-1}$ para cloridrato de piridoxina (VB6), 0,1 a 7,5 $\mathrm{mg} \mathrm{L} \mathrm{L}^{-1}$ cloridrato de tiamina (VB1) e 0,1 a $3,0 \mathrm{mg} \mathrm{L}^{-1}$ para o fosfato

Tabela 1. Valores obtidos nas determinações de vitaminas hidrossolúveis VB1, VB2, VB6 e VPP em amostras comerciais usando-se a técnica CLAE e os declarados nos rótulos ${ }^{a}$ dos medicamentos comerciais

\begin{tabular}{|c|c|c|c|c|}
\hline Amostra & VB1 & VB2 & VB6 & VPP \\
\hline Blepexeron ${ }^{\circledR}$ & $10,1 \pm 0,4$ & $2,5 \pm 0,6$ & $2,4 \pm 0,4$ & $30,2 \pm 0,6$ \\
\hline Blepexeron ${ }^{\circledR}$ & $10,0 \pm 0,5$ & $2,1 \pm 0,6$ & $2,9 \pm 0,5$ & $29,0 \pm 0,6$ \\
\hline${ }^{\text {aBlepexeron }}{ }^{\circledR}$ & 10,0 & 2,5 & 2,5 & 30,0 \\
\hline $\mathrm{EMS}^{\circledR}$ & $30,2 \pm 0,3$ & $10,3 \pm 0,5$ & $10,9 \pm 1,2$ & $20,1 \pm 0,7$ \\
\hline $\mathrm{EMS}^{\circledR}$ & $30,5 \pm 0,3$ & $10,4 \pm 0,6$ & $10,5 \pm 0,7$ & $20,4 \pm 0,9$ \\
\hline${ }^{\mathrm{a}} \mathrm{EMS}^{\circledR}$ & 30,0 & 10,0 & 10,0 & 20,0 \\
\hline Complevitam $^{\circledR}-1$ & $5,1 \pm 0,8$ & $1,2 \pm 0,4$ & $3,0 \pm 0,8$ & $30,3 \pm 0,5$ \\
\hline Complevitam $^{\circledR}-1$ & $5,3 \pm 0,7$ & $1,0 \pm 0,4$ & $3,4 \pm 0,8$ & $30,2 \pm 0,5$ \\
\hline${ }^{\mathrm{a} C}$ Complevitam ${ }^{\circledR}-1$ & 5,0 & 1,0 & 3,0 & 30,0 \\
\hline Suplevit ${ }^{\circledR}$ & $1,2 \pm 0,7$ & $1,1 \pm 0,3$ & $2,2 \pm 0,6$ & $13,1 \pm 0,8$ \\
\hline Suplevit ${ }^{\circledR}$ & $1,3 \pm 0,5$ & $1,0 \pm 0,7$ & $2,0 \pm 0,4$ & $13,3 \pm 0,5$ \\
\hline${ }^{a}$ Suplevit ${ }^{\circledR}$ & 1,3 & 1,3 & 2,0 & 13,0 \\
\hline ComplexoB $^{\circledR}$ & $5,0 \pm 1,4$ & $2,3 \pm 0,9$ & $2,1 \pm 0,9$ & $20,1 \pm 1,3$ \\
\hline ComplexoB $^{\circledR}$ & $5,2 \pm 0,8$ & $2,1 \pm 0,7$ & $2,2 \pm 0,9$ & $20,4 \pm 1,1$ \\
\hline${ }^{\mathrm{a}}$ ComplexoB ${ }^{\circledR}$ & 5,0 & 2,0 & 2,0 & 20,0 \\
\hline de manipulação & $1,5 \pm 0,3$ & $1,4 \pm 0,5$ & $1,9 \pm 0,8$ & $13,2 \pm 1,1$ \\
\hline de manipulação & $1,4 \pm 0,5$ & $1,6 \pm 0,5$ & $2,5 \pm 0,8$ & $14,2 \pm 1,3$ \\
\hline ade manipulação & 1,3 & 1,3 & 2,0 & 13,0 \\
\hline Complevitam $^{\circledR}-2$ & $3,2 \pm 0,5$ & $3,1 \pm 0,7$ & $2,9 \pm 0,5$ & $10,4 \pm 1,0$ \\
\hline Complevitam $^{\circledR}-2$ & $2,8 \pm 0,4$ & $3,2 \pm 0,3$ & $3,2 \pm 0,4$ & $10,6 \pm 1,2$ \\
\hline${ }^{\mathrm{a} C}$ Complevitam ${ }^{\circledR}-2$ & 3,0 & 3,0 & 3,0 & 10,0 \\
\hline
\end{tabular}

Complexo $\mathrm{B}^{\circledR}\left(\mathrm{mg} \mathrm{mL}^{-1}\right)$, Complevitam ${ }^{\circledR}-1\left(\mathrm{mg} 15 \mathrm{~mL}^{-1}\right), \mathrm{EMS}^{\circledR}$ - Complexo B $\left(\mathrm{mg} 10 \mathrm{~mL}^{-1}\right)$, Beplexeron ${ }^{\circledR}\left(\mathrm{mg}^{2,0} \mathrm{~mL}^{-1}\right)$, amostra de manipulação (mg $\left.5 \mathrm{~mL}^{-1}\right)$, Suplevit ${ }^{\circledR}\left(\mathrm{mg} 1,5 \mathrm{~mL}^{-1}\right)$ e Complevitam ${ }^{\circledR}-2\left(\mathrm{mg}^{\circ} \mathrm{mL}^{-1}\right)$.

* Desvio padrão para cinco determinações. 
de riboflavina (VB2). Os dados de concentrações correspondem às variáveis dependentes (y) do processo de calibração.

Para propostas de validação do modelo, sete amostras comerciais do mesmo tipo e nas mesmas faixas de concentrações foram usadas.

\section{Medidas espectrofotométricas na região do ultravioleta}

As 140 amostras obtidas pela diluição da solução estoque de sete amostras comerciais foram analisadas por espectrofotometria na região do ultravioleta. Os espectros foram obtidos em um equipamento UV-Vis Spectrophotometer 2010 Hitachi, equipado com software UV solutions. Os espectros foram adquiridos colocandose cada amostra em uma cubeta de quartzo (10 mm de caminho ótico). Dois espectros de cada amostra foram obtidos e para fins de cálculos foram utilizados os espectros médios. A faixa dos espectros UV utilizados para os cálculos quimiométricos foi de 215 a $314 \mathrm{~nm}$. Os dados dos espectros correspondem às variáveis independentes (x) utilizadas no processo de calibração.

\section{Software}

Os dados foram analisados usando-se o software Matlab versão 6,0 (The Mathworks, Natick, USA) e o toolbox PLS versão 2,0 (Eigenvector Technologies, Manson, USA) deste software.

\section{RESULTADOS E DISCUSSÃO}

\section{Determinação de vitaminas hidrossolúveis B1, B2, B6 e PP usando calibração multivariada e espectrofotometria na região do ultravioleta}

A Figura 1 mostra os espectros na região do UV de amostras de medicamentos e de uma única amostra sintética contendo uma mistura de todas as vitaminas utilizadas neste estudo. Estas amostras foram preparadas por diluições adequadas de soluções estoques de amostras sintéticas e das amostras de medicamentos, em solução tampão acetato $0,2 \mathrm{~mol} \mathrm{~L}^{-1}$ e $\mathrm{pH} 4,0$. As concentrações abrangidas estão dentro da faixa utilizada para as determinações de vitaminas estudadas. De acordo com esta figura é possível verificar que os espectros dos diferentes medicamentos e da amostra sintética são bastante similares. Apesar disso, foi verificado que não é possível empregar amostras sintéticas para a previsão das amostras reais. Os resultados obtidos usando este procedimento (não apresentados neste artigo) são muito insatisfatórios. Esse fato se deve possivelmente à presença de outros componentes presentes nas formulações farmacêuticas que têm influência na determinação (excipientes e, em alguns casos, outros tipos de vitaminas). Amostras sintéticas podem ser utilizadas com resultados satisfatórios em algumas determinações, onde os excipientes podem ser previamente separados. Em outros casos, amostras sintéticas e excipientes podem ser utilizadas na fase de calibração para a obtenção de um modelo mais representativo. Estas duas situações não são aplicáveis neste estudo, devido à grande diversidade dos componentes das formulações farmacêuticas. Neste caso, foi observado que a determinação pode ser apenas realizada usando métodos de calibração multivariada e amostras farmacêuticas comerciais como padrões no modelo de calibração (como realizado em muitos casos de amostras reais).

Neste trabalho, foram utilizados dois diferentes métodos de calibração para verificar sua potencial aplicabilidade na determinação de vitaminas hidrossolúveis em amostras de medicamentos comerciais. Os métodos aqui utilizados foram redes neurais artificiais (RNAs) e mínimos quadrados parciais (PLS).

Os métodos de calibração multivariada baseados em redes

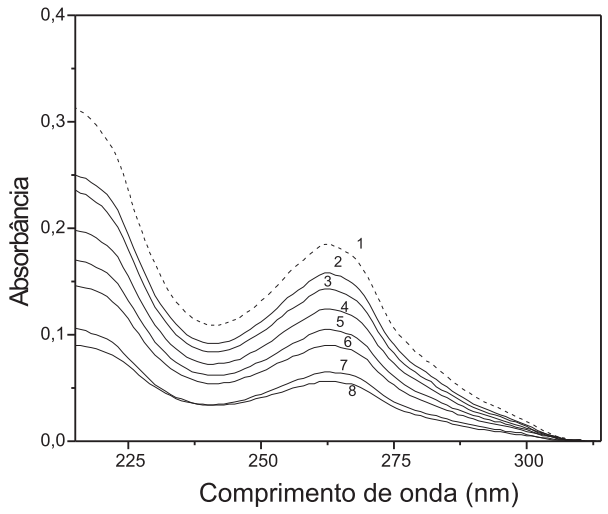

Figura 1. Espectros de absorção na região do ultravioleta de mistura de amostras sintéticas de vitaminas B1, B2, B6 e PP (1) e amostras farmacêuticas:. Complexo $B^{\circledast}(2)$, EMS $^{\varpi}\left(\right.$ complexo B) (3), Beplexeron ${ }^{\circledR}(4)$ amostra de manipulação (5), Suplevit ${ }^{\circledR}$ (6) Complevitam ${ }^{\circledR}-1$ (7) $e$ Complevitam $^{\circledR}-2$ (8). (Condições: tampão acetato 0,2 mol L $^{-1}$ e pH 4,0)

neurais normalmente requerem um grande número de amostras na fase de calibração para que previsões adequadas possam ser obtidas, principalmente quando se tem a presença de amostras complexas. Embora o método de mínimos quadrados parciais (PLS) não requeira um número tão grande de amostras na calibração, neste estudo foi utilizado o mesmo conjunto de calibração empregado para o treinamento de redes neurais. Foi verificado que um número menor de dados não alterava os resultados finais obtidos. Desta forma, manteve-se o mesmo conjunto de dados.

O primeiro modelo adotado, o de mínimos quadrados parciais (PLS) foi construído com os dados obtidos a partir dos espectros na região do UV (variáveis $\mathrm{x}$ ) e os valores das concentrações das vitaminas (variáveis y) baseadas na determinação da concentração das vitaminas, utilizando-se a técnica de cromatografia líquida de alta eficiência (CLAE). Os dados foram dispostos na forma de matrizes: Matriz $\mathbf{X}$ e $\mathbf{Y}$ correspondendo às variáveis $\mathbf{x}$ e $\mathbf{y}$, respectivamente. Modelos específicos foram construídos para cada vitamina, ou seja, os dados de concentrações das vitaminas foram utilizados separadamente para a construção de quatro curvas analíticas. Depois de dispostos na forma de matrizes, os dados foram submetidos a pré-tratamentos matemáticos. No caso do PLS, os dados foram submetidos ao pré-tratamento de centrar na média, que corresponde a fazer com que cada variável tenha média zero. Isto é feito obtendo-se o valor médio para cada variável e subtraindo-o de cada um dos termos da variável correspondente.

O critério para a escolha do número de variáveis latentes que deve ser empregado na calibração foi de validação cruzada. Esta é uma metodologia baseada na magnitude dos erros de previsão de um dado modelo de calibração. De forma geral, o processo é realizado da seguinte maneira: certas amostras são retiradas do conjunto de treinamento usado para a construção do modelo de calibração. A seguir, as amostras que foram retiradas são utilizadas para a previsão, sendo calculados os resíduos referentes a esta previsão. O processo é repetido com outros grupos de amostras, até que todas as amostras tenham sido colocadas fora do modelo de calibração. Calcula-se, então, a soma dos quadrados dos erros de previsão (PRESS). A primeira versão deste método, adotada neste trabalho, consiste em retirar do conjunto de treinamento uma amostra de cada vez. Este método é chamado validação cruzada completa. O processo é repetido com uma, duas e assim por diante, variáveis latentes. Para cada sistema em estudo, o número mais adequado de variáveis latentes será o correspondente ao menor valor de PRESS.

Para a avaliação dos modelos de calibrações obtidos, foram 
empregados cálculos de erro relativo (ER) e de erro quadrático médio de previsão (RMSEP) representado pela Equação 8 .

$R M S E P=\sqrt{\frac{\sum(\text { yreal }- \text { yprev })^{2}}{n}}$

onde yreal são os valores reais para y, yprev são os valores encontrados pelo modelo construído e $n$ é o número de amostras utilizadas.

Para a construção dos modelos de calibração foram utilizadas cinco variáveis latentes para o modelo referente à vitamina B1 (cloridrato de tiamina); para a vitamina B2 foram necessárias quatro variáveis latentes e para a construção dos modelos de calibração para as vitaminas B6 e PP dez variáveis latentes. Pelas análises foi possível verificar que um alto número de variáveis latentes é necessário para a construção de um modelo de calibração para as determinações de vitaminas B6 e PP. Isto pode ser resultado de um pequeno desvio da linearidade dos dados para estas análises devido à presença de outros componentes e, também, à extrema variação nas concentrações destes componentes nas fórmulas farmacêuticas. O modelo de PLS é capaz de fazer boas previsões das concentrações das vitaminas analisadas mesmo em casos onde existe um pequeno desvio da linearidade, adicionando-se mais variáveis latentes para a construção dos modelos de calibração. Devido a esta característica do modelo PLS verifica-se que os resultados apresentados usando este modelo e o de redes neurais não apresentam diferenças significativas quando se emprega um ou outro, embora as redes neurais apresentem resultados relativamente melhores para estas últimas determinações (vitaminas B6 e PP). O mesmo comportamento não é observado para as análises das vitaminas B1 e B2, onde se verificam que os resultados para as análises destas vitaminas são similares utilizando-se ambos os modelos. Neste caso, não existem grandes diferenças nos valores de erros obtidos.

Todos os resultados obtidos para o modelo PLS para a determinação das vitaminas B1, B2, B6 e PP em medicamentos comerciais e a avaliação do desempenho destes modelos baseados em erros relativos e RMSEP são mostrados nas Tabelas 2 e 3 .

$\mathrm{Na}$ aplicação de redes neurais como método de calibração multivariada, os dados também tiveram que passar por pré-tratamentos antes da construção do modelo de calibração. O pré-tratamento matemático utilizado consistiu de subtração dos dados pela média e conseqüente divisão pelo desvio padrão. A seguir, os dados foram reduzidos pela aplicação de análise de componentes principais. Seis componentes principais foram capazes de explicar a variância de 99,9\% dos dados. Os valores de "scores" obtidos pela análise de componentes principais foram utilizados como dados de entrada na primeira camada da rede neural, que é seguida por uma camada interna composta de seis neurônios e uma camada de saída composta

Tabela 2. Resultados obtidos para a determinação de vitaminas hidrossolúveis B1 e B2 em medicamentos comerciais usando-se o método PLS

\begin{tabular}{lcccccc}
\hline Amostras & $\begin{array}{c}\text { HPLC } \\
\text { (VB1) }\end{array}$ & $\begin{array}{c}\text { PLS } \\
\text { (VB1) }\end{array}$ & $\begin{array}{c}\text { ER } \\
(\%)\end{array}$ & $\begin{array}{c}\text { HPLC } \\
\text { (VB2) }\end{array}$ & PLS & $\begin{array}{c}\text { ER } \\
(\%)\end{array}$ \\
\hline Complevitam $^{\circledR}$-1 & 5,1 & 4,9 & 4,0 & 1,2 & 1,1 & $-8,3$ \\
Blepexeron $^{\circledR}$ & 10,1 & 10,3 & 2,0 & 2,5 & 2,6 & 4,0 \\
EMS $^{\circledR}$ & 30,2 & 29,0 & $-4,0$ & 10,3 & 9,8 & $-4,9$ \\
ComplexoB $^{\circledR}$ & 5,2 & 5,2 & 0 & 2,1 & 2,2 & 4,5 \\
de manipulação & 1,5 & 1,4 & $-6,7$ & 1,4 & 1,5 & 6,7 \\
Suplevit $^{\circledR}$ & 1,3 & 1,4 & 7,7 & 1,0 & 1,1 & 10,0 \\
Complevitam $^{\circledR}-2$ & 2,8 & 3,0 & 7,1 & 3,2 & 3,3 & 3,1 \\
RMSEP* $^{*}$ & & & $5,2 *$ & & & $6,4 *$ \\
\hline
\end{tabular}

Tabela 3. Resultados obtidos para a determinação de vitaminas hidrossolúveis B6 e PP em amostras farmacêuticas comerciais usandose o método de PLS

\begin{tabular}{lcccccc}
\hline Amostras & $\begin{array}{c}\text { HPLC } \\
\text { (VB6) }\end{array}$ & $\begin{array}{c}\text { PLS } \\
\text { (VB6) }\end{array}$ & $\begin{array}{c}\text { ER } \\
(\%)\end{array}$ & $\begin{array}{c}\text { HPLC } \\
\text { (VPP) }\end{array}$ & PLS & $\begin{array}{c}\text { ER } \\
(\%)\end{array}$ \\
\hline Complevitam $^{\circledR}-1$ & 3,0 & 3,2 & 6,7 & 30,3 & 30,3 & 0 \\
Blepexaron $^{\circledR}$ & 2,4 & 2,5 & 4,2 & 30,2 & 30,4 & 0,7 \\
EMS $^{\circledR}$ & 10,9 & 10,6 & $-2,8$ & 20,1 & 20,2 & 0,5 \\
ComplexoB $^{\circledR}$ & 2,1 & 2,2 & 4,7 & 20,4 & 20,4 & 0 \\
de manipulação $^{\text {Suplevit }}{ }^{\circledR}$ & 1,9 & 1,7 & $-10,5$ & 13,2 & 13,8 & 4,5 \\
Complevitam $^{\circledR}-2$ & 2,0 & 2,2 & $-10,0$ & 13,3 & 13,5 & 1,5 \\
RMSEP & 3,2 & 3,3 & 3,1 & 10,6 & 11,0 & 3,8 \\
& & & $6,7 *$ & & & $2,3 *$ \\
\hline
\end{tabular}

de um neurônio. Esta foi a arquitetura da rede neural utilizada neste trabalho. Uma função sigmoidal foi utilizada na camada interna como função de transferência e uma função linear foi usada na camada de saída. Para o treinamento da rede neural, o algoritmo de MarquardtLevenberg foi empregado. No treinamento da rede foram realizados 300 processos de iterações, sendo utilizado como critério de completo treinamento da rede a obtenção de um valor de erro para o modelo de calibração correspondendo a $1 \times 10^{-2}$. Os resultados correspondentes aos modelos de redes neurais, bem como os valores de erro relativo e RMSEP utilizados como critérios para a avaliação destes modelos, são apresentados na Tabela 4 para as determinações das vitaminas B1 e B2 e na Tabela 5 para as determinações de VB6 e VPP, respectivamente.

Tabela 4. Resultados obtidos para a determinação de vitaminas hidrossolúveis B1 e B2 em amostras farmacêuticas comerciais usando-se ANN

\begin{tabular}{|c|c|c|c|c|c|c|}
\hline Amostras & $\begin{array}{l}\text { HPLC } \\
\text { (VB1) }\end{array}$ & $\begin{array}{l}\text { RNA } \\
\text { (VB1) }\end{array}$ & $\begin{array}{l}\text { ER } \\
(\%)\end{array}$ & $\begin{array}{l}\text { HPLC } \\
\text { (VB2) }\end{array}$ & $\begin{array}{l}\text { RNA } \\
\text { (VB2) }\end{array}$ & $\begin{array}{l}\text { ER } \\
(\%)\end{array}$ \\
\hline Complevitam $^{\circledR}-1$ & 5,1 & 5,3 & 4,0 & 1,2 & 1,1 & $-9,8$ \\
\hline Blepexeron $^{\circledR}$ & 10,1 & 10,6 & 5,0 & 2,5 & 2,5 & 0 \\
\hline $\mathrm{EMS}^{\circledR}$ & 30,2 & 30,4 & 0,7 & 10,3 & 10,3 & 0 \\
\hline ComplexoB ${ }^{\circledast}$ & 5,2 & 5,3 & 1,9 & 2,1 & 2,3 & 9,5 \\
\hline de manipulação & 1,5 & 1,6 & 6,7 & 1,4 & 1,5 & 7,1 \\
\hline Suplevit $^{\circledR}$ & 1,3 & 1,4 & 7,7 & 1,0 & 1,0 & 0 \\
\hline Complevitam $^{\circledR}-2$ & 2,8 & 3,0 & 7,1 & 3,2 & 3,1 & $-3,1$ \\
\hline RMSEP* & & & $5,3^{*}$ & & & $6,0 *$ \\
\hline
\end{tabular}

Tabela 5. Resultados obtidos para a determinação de vitaminas hidrossolúveis B6 e PP em amostras farmacêuticas comerciais usando-se RNA

\begin{tabular}{lcccccc}
\hline Amostras & $\begin{array}{c}\text { HPLC } \\
\text { (VB6) }\end{array}$ & $\begin{array}{c}\text { RNA } \\
\text { (VB6) }\end{array}$ & $\begin{array}{c}\text { ER } \\
(\%)\end{array}$ & $\begin{array}{c}\text { HPLC } \\
\text { (VPP) }\end{array}$ & RPA & $\begin{array}{c}\text { ER } \\
(\%)\end{array}$ \\
\hline Complevitam $^{\circledR}-1$ & 3,0 & 3,1 & 3,3 & 30,3 & 30,4 & $-0,3$ \\
Blepexeron $^{\circledR}$ & 2,4 & 2,5 & 4,2 & 30,2 & 30,5 & 0,9 \\
EMS $^{\circledR}$ & 10,9 & 10,7 & $-1,9$ & 20,1 & 20,5 & 2,0 \\
ComplexoB $^{\circledR}$ & 2,1 & 2,3 & 9,5 & 20,4 & 20,3 & $-0,5$ \\
de manipulação & 1,9 & 2,0 & 5,3 & 13,2 & 13,2 & 0 \\
Suplevit $^{\circledR}$ & 2,0 & 2,1 & 5,0 & 13,3 & 13,3 & 0 \\
Complevitam $^{\circledR}-2$ & 3,2 & 3,2 & 0 & 10,6 & 10,5 & $-0,9$ \\
RMSEP $^{*}$ & & & $5,0 *$ & & & $0,9 *$ \\
\hline
\end{tabular}




\section{CONCLUSÃO}

Os métodos de calibração multivariada baseados em mínimos quadrados parciais (PLS) e redes neurais artificiais (RNAs) em conjunto com a técnica de espectrofotometria na região do ultravioleta podem ser empregados para a determinação de vitaminas hidrossolúveis VB1, VB2, VB6 e VPP em amostras complexas de medicamentos comerciais. Em todos os casos foram obtidos bons resultados para a determinação de vitaminas hidrossolúveis nos medicamentos. Verifica-se que o método PLS pode modelar adequadamente os dados, mesmo em presença de um pequeno desvio da linearidade (verificado para as determinações de VB6 e VPP).

Estes métodos são simples e rápidos e podem substituir os métodos tradicionalmente empregados para a determinação de vitaminas em amostras comerciais, podendo se constituir em um instrumento eficaz em análises de rotina para o controle de qualidade destes produtos.

\section{REFERÊNCIAS}

1. Kirk-Othmer Encyclopedia of chemical technology, A Wiley-Interscience publication, John Wiley \& Sons: New York, 1984, p. 24
2. Rizollo, A.; Polesello, S.; J. Chromatogr. 1992, 624, 103

3. Hollman, P. C. H.; Slanger, J. H.; Wagstafe, P. J.; Faured, U.; Southgate, A. A. T.; Finglas, P. M.; Analyst 1993, 118, 481

4. Agostini, T. S.; Godoy, H. T.; J. High Resol. Chromatogr. 1997, $20,245$.

5. Verma, K. K.; Jain, A.; Sahasrabuddhey, B.; Gupta, K.; Mishira, S.; J. AOCS 1996, 79, 1236.

6. Wu, H.; Oguma, K.; Yu, R. Q.; Anal. Sci. 1994, 10, 875

7. Wang, J.; Han, J.; Su, B.; Lin, C.; Wamg, N.; Hu, J.; Anal.Sci. 1998, 14, 965.

8. Lopes-de-Alba, P. L.; Lopes-Martinez, L.; Cerda, V.; Amador-Hernández, J.; J. Braz. Chem. Soc. 2006, 17, 715 .

9. Abeásturi, F. J.; Jiménez, I.; Arias, J. J.; Jiménez, F.; Anal. Lett. 2002, 35, 1677.

10. Hernández, S. R.; Ribeiro, G. G.; Goicoechea, H. C.; Talanta 2003, 61, 743.

11. Ferreira, M. M. C.; Antunes, A. M.; Melgo, M. S.; Volpe, P. L. O.; Quim. Nova 1999, 22, 724

12. Martens, H.; Naes, T.; Multivariate Calibration, John Wiley \& Sons: New York, 1989.

13. Wise, B. M.; Gallagher, N. B.; PLS-Toolbox 2.1 for use with Matlab EigenVector Research Inc: Manson, 1998.

14. Zampronio, C. G.; Rohweder, J. J. R.; Poppi , R. J.; Chemom. Intell. Lab. Syst. 2002, 62, 17.

15. Despagne, F.; Massart, D. L.; Analyst 1998, 123, 157R.

16. Demuth, H.; Beale, M.; Neural Network toolbox for use with Matlab, EigenVector Research Inc: Manson, 1998. 